\title{
Estimation of Arterial Blood Pressure Based on Artificial Intelligence Using Single Earlobe Photoplethysmography during Cardiopulmonary Resuscitation
}

\author{
Jong-Uk Park ${ }^{1} \cdot$ Dong-Won Kang ${ }^{1,2} \cdot$ Urtnasan Erdenebayar $^{1} \cdot$ Yoon-Ji Kim ${ }^{1} \cdot$ Kyoung-Chul Cha $^{3}$. \\ Kyoung-Joung Lee ${ }^{1}$ (i)
}

Received: 6 October 2019 / Accepted: 26 November 2019 / Published online: 10 December 2019

(C) Springer Science+Business Media, LLC, part of Springer Nature 2019

\begin{abstract}
This study investigates the feasibility of estimation of blood pressure (BP) using a single earlobe photoplethysmography (Ear PPG) during cardiopulmonary resuscitation (CPR). We have designed a system that carries out Ear PPG for estimation of BP. In particular, the BP signals are estimated according to a long short-term memory (LSTM) model using an Ear PPG. To investigate the proposed method, two statistical analyses were conducted for comparison between BP measured by the micromanometer-based gold standard method ( $\mathrm{BP}_{\mathrm{MEAS}}$ ) and the Ear PPG-based proposed method $\left(\mathrm{BP}_{\mathrm{EST}}\right)$ for swine cardiac model. First, Pearson's correlation analysis showed high positive correlations $(r=0.92, p<0.01)$ between $\mathrm{BP}_{\mathrm{MEAS}}$ and $\mathrm{BP}_{\mathrm{EST}}$. Second, the paired-samples $t$-test on the $\mathrm{BP}$ parameters (systolic and diastolic blood pressure) of the two methods indicated no significant differences $(p>0.05)$. Therefore, the proposed method has the potential for estimation of BP for CPR biofeedback based on LSTM using a single Ear PPG.
\end{abstract}

Keywords Cardiopulmonary resuscitation (CPR) · Biofeedback · Earlobe photoplethysmography (ear PPG) · Blood pressure (BP) $\cdot$ Long short-term memory (LSTM)

\section{Introduction}

Cardiopulmonary resuscitation (CPR) is an emergency procedure to maintain brain function by supplying blood flow to the brain in patients under cardiac arrest [1]. It is important to correctly perform CPR to increase the survival rate of such patients [1]. However, even well-trained CPR professionals fail to consistently act in accordance with the standards of the American Heart Association (AHA) guidelines because each patient has different physical characteristics. [2]. Therefore, a CPR feedback system is necessary to monitor

Jong-Uk Park and Dong-Won Kang contributed equally to this work.

This article is part of the Topical Collection on Mobile \& Wireless Health

Kyoung-Joung Lee

1kj5809@yonsei.ac.kr

1 Department of Biomedical Engineering, College of Health Science, Yonsei University, Wonju, Kangwon-do 26493, South Korea

2 R\&D Center, MEDIANA Co., Ltd., Wonju, Republic of Korea

3 Department of Emergency Medicine, Wonju College of Medicine, Yonsei University, Wonju, Republic of Korea the condition of cardiac-arrest patients during CPR for the sake of its efficient realization.

The gold standard for circulation detection is invasive blood pressure (BP) during CPR. However, it is only feasible at the time in-hospital [3]. The AHA guidelines recommend using a capnogram as a support tool to decide about the return of spontaneous circulation (ROSC) [4]. An increase in endtidal carbon dioxide $\left(\mathrm{ETCO}_{2}\right)$ level may serve as an indicator of ROSC [3]. However, a capnogram is only available with intubation and has low accuracy in ROSC detection [3]. Researchers have proposed CPR feedback methods using a transthoracic-thoracic impedance (TTI) [5] or accelerometers [6] to efficiently perform CPR. However, these methods have some limitations such as uncomfortable, time-consuming, or inaccurate procedures. Especially, it is not exactly known whether blood is delivered to the brain during CPR.

Therefore, we propose a new method for CPR feedback based on artificial intelligence using earlobe photoplethysmography (Ear PPG) in this study. Ear PPG is noninvasive and has the advantage of reflecting the blood flow to brain in real time. Besides, BP can be estimated according to a long short-term memory (LSTM) model, which is an artificial intelligence structure, using Ear PPG. 


\section{Materials \& Methods}

Data were collected from two swines $(70-75 \mathrm{~kg})$ with cardiac-arrest model after approval by the Institutional Animal Care and Use Committee of Wonju College of Medicine, Yonsei University, for this study. Subjects were placed in a supine position and ventilated using a volumecontrolled ventilator (MDS Matrix 3000, Matrix Environmental Technologies Inc. USA) after anesthesia. Electrocardiography (Lead II ECG), ETCO $_{2}$ (CO2SMO plus, Novametric Medical Systems Inc. USA) and atrial blood pressure (5-Fr micromanometer-tipped catheter, Millar Inc. USA) were continuously monitored using a digital recording system (PowerLab, ADInstruments Ltd. USA). A pacing catheter was positioned in the right ventricle and ventricular fibrillation (VF) and asystole (Asys) was induced by delivering an alternating electrical current at $60 \mathrm{~Hz}$ with $60 \mathrm{~V}$ and $30 \mathrm{~mA}$ to the endocardium. CPR was performed according to the sequence rest (10 s) - CPR (40 s) - rest (10 s). An active compression-decompression (ACD) CPR device (LUCAS2, Physio-Control CO, USA) was used to achieve an uniform chest compression depth and compression rate, which were set at $5 \mathrm{~cm}$ and $100 / \mathrm{m}$, respectively. To develop and evaluate the proposed method, a total of 255 datasets were randomly divided as follows: 135 datasets formed a training set (VF/Asys: 87/48) and 80 datasets formed a test set (VF/Asys: 58/32).

Figure 1 shows the method of BP estimation using Ear PPG based on LSTM model for CPR feedback. The analog circuit of the proposed system consists of an earlobe cliptype PPG sensor (EP520, LAXTHA Inc., Republic of Korea), an analog filter and an amplifier. The earlobe PPG sensor is composed of a visible red emitter with a center wavelength of $630 \mathrm{~nm}$, a phototransistor, and an I-V converter. It enables the measurement of changes of blood volume caused by CPR. The PPG signals were amplified with a gain of 100 and filtered with a $0.15-20 \mathrm{~Hz}$ band-pass filter to remove power noise and baseline variation. Then, the data were digitized and saved at $10 \mathrm{bits} / \mathrm{sample}$ and 360 samples/s using PIC18F4523 (Microchip Technology Inc., USA). The acquired data were first filtered using a digital band-pass filter of bandwidth $0.5-10 \mathrm{~Hz}$ and then resampled to $20 \mathrm{~Hz}$. The resulting signal is used as the input for the LSTM model. The LSTM architecture to estimate the BP waveform consists of three layers, namely an input layer, a hidden layer, and an output layer. To determine the optimal number of hidden layers, they were added from one to seven, step by step, while interactively evaluating the performance of each model. The best performance was obtained when using one hidden layer. Finally, two BP parameters (systolic and diastolic blood pressure) were calculated.

\section{Results}

Table 1 shows the results of statistical analyses for comparison between BP measured by the micromanometer-based gold standard method ( $\mathrm{BP}_{\mathrm{MEAS}}$ ) and the proposed Ear PPG-based

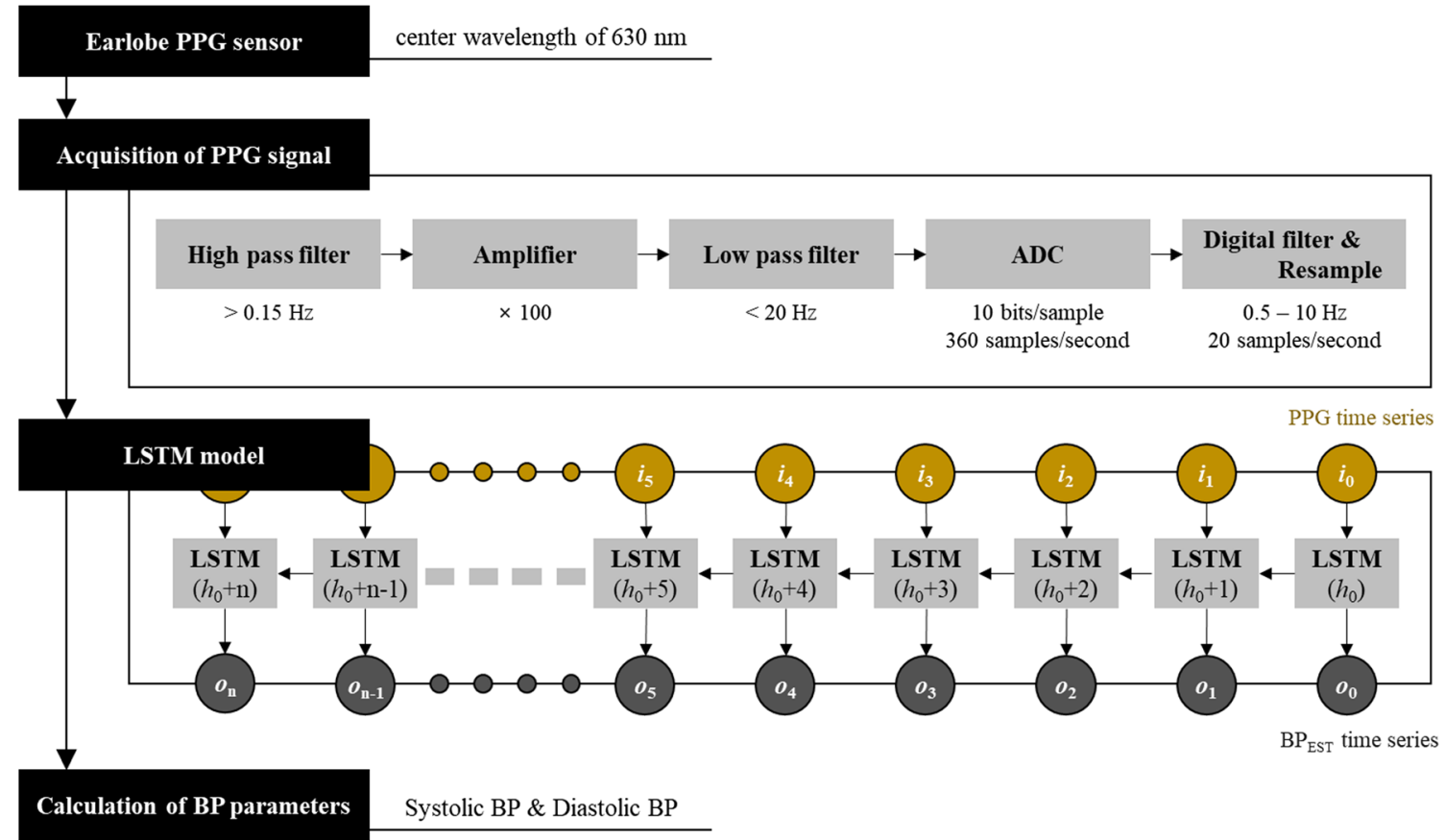

Fig. 1 Block diagram of BP estimation using Ear PPG based on LSTM model for CPR feedback 
Table 1 Results of statistical analyses for comparison between BP measured by the micromanometer-based gold standard method and the Ear PPGbased proposed method during CPR

\begin{tabular}{llll}
\hline Training set $(\boldsymbol{N}=\mathbf{1 3 5})$ & Pearson's correlation $[r(p$ value $)]$ & Paired-samples $t$-test $[p$ value $]$ & RMSE $[\mathrm{mean} \pm \mathrm{SD}]$ \\
\hline BP waveform & $0.93(<0.01)$ & - & - \\
Systolic blood pressure & - & 0.17 & $2.10 \pm 1.15 \mathrm{mmHg}$ \\
Diastolic blood pressure & - & 0.06 & $1.88 \pm 1.19 \mathrm{mmHg}$ \\
Test set $(\boldsymbol{N}=\mathbf{8 0})$ & Pearson's correlation $[r(p$ value $)]$ & Paired-samples $t$-test $[p$ value $]$ & $\mathrm{RMSE}[\mathrm{mean} \pm \mathrm{SD}]$ \\
BP waveform & $0.92(<0.01)$ & - & - \\
Systolic blood pressure & - & 0.18 & $2.24 \pm 1.37 \mathrm{mmHg}$ \\
Diastolic blood pressure & - & 0.06 & $1.90 \pm 1.20 \mathrm{mmHg}$ \\
\hline
\end{tabular}

RMSE: root mean-squared error, SD: standard deviation

method $\left(\mathrm{BP}_{\mathrm{EST}}\right)$ during $\mathrm{CPR}$. Pearson's correlation analysis showed high positive correlations $(r=0.92, p<0.01)$ between

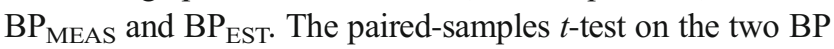
parameters of the two methods indicated no significant differences $(p>0.05)$. The root mean-squared error (RMSE) of the systolic and diastolic BP was $2.24 \pm 1.37 \mathrm{mmHg}$ and $1.90 \pm$ $1.20 \mathrm{mmHg}$, respectively.

\section{Discussion \& Conclusion}

This study proposes single Earlobe PPG-based BP estimation during CPR. We designed the analog circuit to acquire PPG signals during CPR and the LSTM model to estimate the BP waveform. Then, statistical analyses were conducted for comparison between BP measured by the gold standard and the proposed method. The estimated BP showed high positive correlations with the measured BP. The RMSE of the calculated systolic and diastolic BP was $2.24 \pm 1.37 \mathrm{mmHg}$ and $1.90 \pm 1.20 \mathrm{mmHg}$, respectively.

Zadi et al. proposed an auto-regressive moving average (ARMA) model for a BP estimation algorithm using a PPG, resulting in an RMSE of 3-8 $\mathrm{mmHg}$ in young subjects with no known cardiovascular disorder [7]. Tanveer et al. estimated BP from an ECG and a PPG using a waveform-based ANN-LSTM network. This method achieved a high performance, namely $0.58-1.56 \mathrm{mmHg}$ in multi parameter intelligent monitoring in intensive care (MIMIC) I database [8]. The performance of our study is similar or slightly lower compared with these studies. However, the strength in our study is that BP estimation is possible even during CPR. It enables accurate CPR, thereby increasing ROSC in patients under cardiac arrest. Therefore, we are convinced that the proposed method can be used as an important and useful tool to provide accurate CPR. In a further study, we will test for more subjects and patients under cardiac arrest to ensure the reliability of this method and optimize the LSTM model to improve performance.

\section{Compliance with Ethical Standards}

Conflict of Interest All authors declare that they have no conflict of interest.

Ethical Approval All procedures performed in studies involving human participants were in accordance with the ethical standards of the institutional and/or national research committee and with the 1964 Helsinki declaration and its later amendments or comparable ethical standards.

Informed Consent Informed consent was obtained from all individual participants included in this study.

\section{References}

1. Sakabe, T., Tateishi, A., Miyauchi, Y., Maekawa, T., Matsumoto, M., Tsutsui, T., and Takeshita, H., Intracranial pressure following cardiopulmonary resuscitation. Intensive Care Med 13(4):256-259, 1987. https://doi.org/10.1007/BF00265114.

2. Cheng, A., Brown, L. L., Duff, J. P. et al., Improving cardiopulmonary resuscitation with a $\mathrm{CPR}$ feedback device and refresher simulations (CPR CARES study): A randomized clinical trial. JAMA Pediatr 169(2):137-144, 2015. https://doi.org/10.1001/ jamapediatrics.2014.2616.

3. Wei, L., Chen, G., Yang, Z. et al., Detection of spontaneous pulse using the acceleration signals acquired from CPR feedback sensor in a porcine model of cardiac arrest. PLoS ONE 12(12):e0189217, 2017. https://doi.org/10.1371/journal.pone.0189217.

4. Callaway, C. W., Soar, J., Aibiki, M. et al., Part 4: Advanced life support: 2015 international consensus on cardiopulmonary resuscitation and emergency cardiovascular care science with treatment recommendations. Circulation 132(16_supp1_1):S84-S145, 2015. https://doi.org/10.1016/j.resuscitation.2015.07.042.

5. Berve, P. O., Irusta, U., Kramer-Johansen, J. et al., Transthoracic impedance measured with defibrillator pads- New interpretations of signal change induced by ventilations. J Clin Med 8(5):724, 2019. https://doi.org/10.3390/jcm8050724.

6. Gruber, J., Stumpf, D., Zapletal, B. et al., Real-time feedback systems in CPR. Trends Anaesth Crit Care 2(6):287-294, 2012. https:// doi.org/10.1016/j.tacc.2012.09.004. 
7. Zadi, A. S., Alex, R., Zhang, R. et al., Arterial blood pressure feature estimation using photoplethysmography. Comput Bio Med 102: 104-111, 2018. https://doi.org/10.1016/j.compbiomed.2018.09.013.

8. Tanveer, M. S., and Hasan, M. K., Cuffless blood pressure estimation from electrocardiogram and photoplethysmogram using waveform based ANN-LSTM network. Biomed Sig Process Control 51:382392, 2019. https://doi.org/10.1016/j.bspc.2019.02.028.

Publisher's Note Springer Nature remains neutral with regard to jurisdictional claims in published maps and institutional affiliations. 